TANia PÉrez Bustos*

MANUEL FRANCO**

\title{
SOBRE LA EDUCACIÓN NO FORMAL, EL UNIVERSO TECNOLÓGICO Y EL GOCE
}

\begin{abstract}
La principal razón para educar personas que se dediquen a la ciencia (y la tecnologia) tiene que ver con la democracia. De otra forma, si uno no entiende el mundo que lo rodea siente miedo.
\end{abstract}

Roald Hoffmann

\begin{abstract}
Resumen
En el presente documento describimos la experiencia de Maloka, un centro interactivo de ciencia y tecnología, en cuanto a generación de clubes de tecnología como escenarios para el manejo y aprovechamiento del tiempo libre se refiere. Hacemos énfasis de manera particular en la propuesta metodológica que hemos desarrollado y que tiene como uno de sus pilares fundamentales el goce entendido como motivación. Este trabajo, desarrollado en los clubes de tecnologia, está altamente influenciado por las transformaciones que la revolución electrónica ha introducido en el nuevo mundo tecnocultural, en el que los flujos de információn y la abundancia de componentes electrónicos, inundan la cotidianidad del individuo y lo convierten en su interlocutor gracias sólo a su utilización. Es desdo ol reconocimiento de este contexto que planteamos una manera de acercarse al público, ofreciéndole una visión de la tecnología que atiende a sus expectativas y que responde con una postura crítica frente a ella, apostándole a la formación de ciudadanos capaces de crear y repensar su universo, apropiarse y entender la tecnología.
\end{abstract}

Palabras clave: Museos, educación en tecnología, diálogo de saberes.

\section{THE REJOICE, THE TECHNOLOGICAL UNIVERSE} AND THE NO FORMAL EDUCATION

\section{Abstract}

This document describes the experience of Maloka, an interactive center for science and technology, creating technology clubs as a strategy to promote the management and productive use of free time. Particularly, the paper emphasizes in the methodological proposal that has been developed by the Maloka research team. This proposal has the concept of entertainment, as a motivational tool, as one fo its fundamental pillars. This work in the technology clubs is highly influenced by the transformations that the electronic revolution has introduced in a new tecnocultural

* Antropóloga, comunicadora social, magister en estudios sobre el desarrollo. Coordinadora de sistematización e investigación del Centro Interactivo de Ciencia y Tecnologia (Maloka). tbustos@yahoo.com.

** Ingeniero mecánico. Especialista en pedagogía. Jefe de desarrollo y producción, y coordinador de los Clubes de Tecnologia de Maloka. (Bogotá). mfrancoavellaneda@yahoo.com.

Texto recibido el 19 de agosto y aprobado el 3 de noviembre de 2005.

Pedagogía y Saberes N.ํㅜㄹ. 23. Universidad Pedagógica Nacional. Facultad de Educación, 2005, pp. 103-110 
World: flooding quotidian life with information and electronic components and transforming the subject in a user of technology. The methodological proposal developed in Maloka does recognize this context of changes, listening to the public expectations related to technology but looking forward to construct with them a critical vision of the technological World, and developing as it goes a new generation of citizens capable of creating and re-thinking their World through the understanding of technology.

Key words: museums, education for technology, entertainment, knowledges dialogues.

La relación entre el goce y la educación en el territorio de los escenarios culturales, como los museos, es una relación, en muchos sentidos, polifónica, que busca desde seducir hasta convencer, pasando por la curiosidad, el disfrute y el conocimiento (Sautiño, 2005). Para entender esta relación es importante enmarcar el contexto en el cual los museos son entendidos como lugares en los que el entretenimiento es posible y vislumbrar allí cómo esta perspectiva define un norte para estos escenarios, orientando su misión educativa hacia horizontes que no siempre son contemplados institucionalmente. Este artículo pretende hacer un acercamiento a esta reflexión dando cuenta de la experiencia de Maloka, un centro interactivo de ciencia y tecnología en Colombia ${ }^{1}$, en donde se ha construido una metodología para la educación no formal en tecnologia, que entiende el goce como una estrategia pilar de su propuesta educativa.

\section{EL GOCE Y SU SENTIDO PRODUCTIVO}

Sin negar que la misión que un parque de diversiones cumple es ontológicamente distinta a la que cumple un centro de ciencia y tecnologia, hay que reconocer que dentro de un entorno urbano ambos escenarios tienen una característica común: son lugares reconocidos por los habitantes de la ciudad como ofertas alternativas para manejar

${ }^{1}$ Para mayor información sobre el museo visitar www.maloka.org

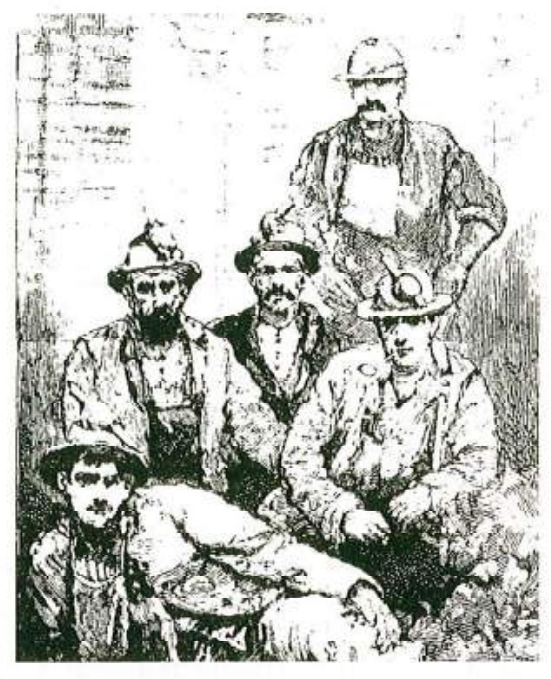

Esta concepción del público con relación al para qué del museo enfrenta a la institución con la necesidad de repensar el cómo de su misión divulgadora o de apropiación social. Tradicionalmente los escenarios culturales se han entendido como lugares para dar a conocer la ciencia, el arte y la vida, entre otras cosas (Cremers, 2003: 21-26). A partir de esta concepción, la relación que el museo mantiene con sus públicos puede concebirse desde una perspectiva comunicativa muy lineal: el museo comunica un mensaje que su público recibe y que quizás no atienda a la demanda cultural en la que está inscrito. El hecho que como escenario cultural sea reconocido como un lugar para el entretenimiento lleva a que sea importante explorar, por un lado, cómo la apropiación social de la tecnologia ${ }^{2}$ puede entretener, y por otro, cómo el entretenimiento puede tener un sentido de apropiación social.

es una sociedad que contempla el goce con una connotación productiva, es decir, que se entretiene conociendo, explorando, descubriendo y aprehendiendo el mundo.

Esta idea de disfrute está directamente ligada al aprendizaje lúdico, ya que se convierte en un puente que permite acercar la ciencia y la tecnología al público en general; en otras palabras, es simultáneamente un fin social y una herramienta constructiva; un fin en la medida en que elimina prejuicios sobre el conocimiento científico-tecnológico permitiendo que éste sea abordado de modo más natural; y una herramienta, puesto que nos da la oportunidad de encontrarnos con el mundo en nuevas dimensiones, resignificándolo.
En esta búsqueda por generar una tiva y menos centrada en los objetos (Gagliardi, 2005), es decir, por repensar estratégicamente su misión de apropiación social de la ciencia y la tecnologia, desde Maloka se han generado los clubes de tecnología como una propuesta que atiende a la lógica del goce productivo en tanto estrategia o finalidad metodológica de la educación no formal.

${ }^{2}$ Este es el caso de Maloka y de los clubes de tecnologia de manera particular; sin embargo, no deja de ser una pregunta válida para contextos artísticos, cientificos o culturales. relación con el público más interac- 
Los clubes de tecnologia de Maloka tienen hasta la fecha tres años de existencia y se han constituido como un espacio para que los niños, entre los nueve y los catorce años de edad, tengan la posibilidad de reconocer su universo tecnológico a través de experiencias didácticas que responden a una pedagogía crítica, según la cual, reconocer y considerar prácticamente los referentes tecnológicos cotidianos de los participantes es algo fundamental para que puedan explorar de manera creativa los mundos de la energía, el aire y el vuelo, y la robótica ${ }^{3}$.

Es de anotar que los niños que participan de estos escenarios lo hacen de manera libre (no son forzados a participar y deciden su asistencia a las sesiones), visitando el centro interactivo durante quince fines de semana por semestre $y$, en algunos casos, durante periodos de vacaciones escolares. En este contexto, los clubes de tecnologia de Maloka se inscriben como escenarios para el aprovechamiento del tiempo libre y son reconocidos por sus participantes como lugares para explorar el mundo de la tecnologia de una manera lúdi$\mathrm{ca}^{4}$, entendiendo lo lúdico desde lo que hemos denominado aquí como la posibilidad de gozar productivamente de un espacio (Mendia, 1991: 1-2); por último, los clubes se constituyen

${ }^{3}$ En este articulo vamos a hacer énfasis particular en el club de robótica, que cuenta con mayor antigüedad y en el que es más evidente la relación mediática del público con el tema y, por tanto, se hace más clara la propuesta metodológica relacionada con el goce, la cual es de interés para esta publicación.

${ }^{4} \mathrm{En}$ el contexto de la investigación realizada por el Insitituto Colombiano para el Desarrollo de la Ciencia y la Tenologia, Conciencias, sobre la percepción y apropiación social de la ciencia en Colombia, se ha señalado que el $88 \%$ de los docentes de educación básica y media considera que Maloka es el lugar para el aprendizaje lúdico más reconocido por niños y jóvenes (Abud, et al, 2005: 14). en una estrategia de apropiación social que promueve tres elementos fundamentales: en primer lugar, el diálogo de saberes tecnológicos entre los tutores jóvenes (profesionales de áreas técnicas en formación $^{5}$ ) de los clubes y los niños que participan en él (Ghiso, 2000: 2); en segundo término, la posibilidad de que los participantes se acerquen a la manipulación práctica y crítica de disciplinas duras como la mecánica, la electrónica y la informática, que son parte del universo de la robótica (Rodríguez, 1996: 5); por último, la reflexión de cada niño sobre su experiencia tecnológica vivida cada fin de semana (McLaren, 1998: 74-75).

Vamos ahora a profundizar sobre las razones concretas que hacen que un escenario con estas finalidades de apropiación social descritas, sea reconocido como un escenario para "pasarla bien" o para que niños se entretengan. Para ello nos concentraremos en el club de robótica.

\section{EL GOCE DE SER GRANDE. ENTRE DISCOVERY CHANNEL Y STAR WARS}

La innovación tecnológica y científica es característica de la sociedad del siglo XXI, entendiendo innovación como la apropiación cultural de los nuevos desarrollos de las nuevas generaciones (Medina, 2003: 52-53. 59). De esta manera, lo que sucede entre la robótica y los niños es un acto de seducción y encantamiento en el que tienen mucho que ver los medios masivos de comunicación. Los niños saben sobre este universo gracias a lo que han visto en Discovery Channel o se imaginan su potencial, pues lo comparan con la ciencia ficción de películas. como Star Wars. En otras palabras,

${ }^{5}$ La mayoria de los tutores son estudiantes de carreras ingeniería, en el equipo hay, entre otros, estudiantes de ingenieria de sistemas, electrónica, mecatrónica y mecánica. su posición frente al universo de la robótica es una mezcla de realidad e imaginación que no siempre se acerca a su contexto cotidiano. La robótica es para los niños algo mági$\mathrm{co}$, algo que existe y que les permite construir el mundo a su medida, pero cuyo origen es un completo misterio (Giddens, 1994: 32 y ss.). Este es un umbral simbólico que marca las expectativas de los niños al entrar al club. Según Jesús Martin-Barbero, es un ejemplo de cómo se invita al niño a participar del mundo real de los adultos a través de los medios (Martín-Barbero, 1998). En este acercamiento mediático se inserta una idea de goce relacionado con la actividad lúdica, a través del cual se hace manifiesto el deseo de los niños de ser incluidos socialmente (Bautista-Vallejo, 2002: 3).

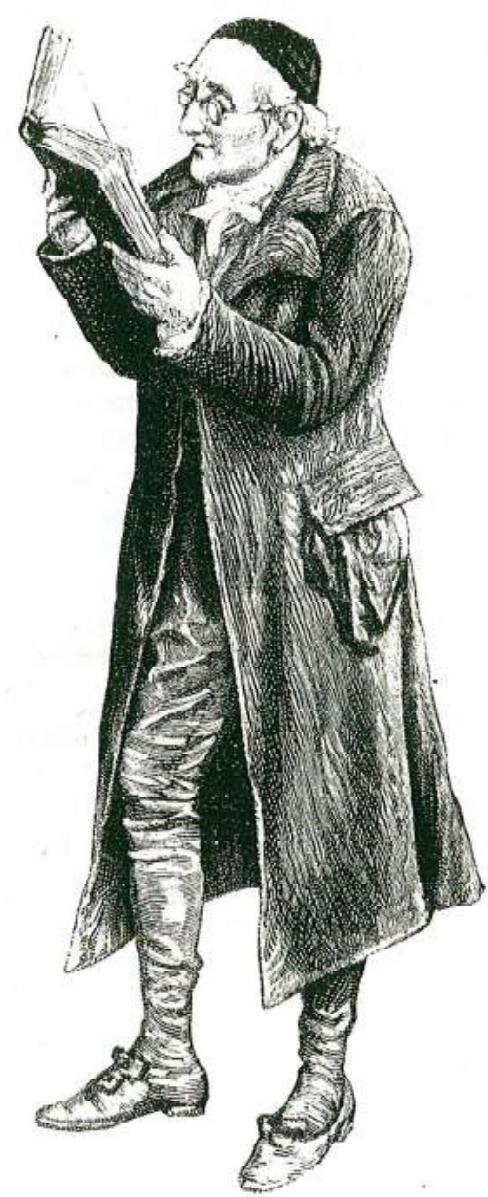


En este sentido, la relación entre los clubes de tecnología y su público potencial es una relación que de algún modo está "mediada", es decir, que genera las expectativas de que lo que muestran los medios se puede vivir en los clubes. Así las cosas, los clubes se convierten para los niños en una respuesta concreta, real, asible a los imaginarios generados por la televisión y el cine. Los niños van a Maloka a construir un r2d2 o un c3po ${ }^{6}$, a desarrollar una máquina capaz de hacer cosas por ellos o cosas como las que ellos son capaces de hacer. Prueba de esto son las imágenes que los participantes del club construyen de lo que un robot es al inicio de la primera sesión. Bajo la premisa de que uno de los objetivos de principio del club de robótica es construir un robot (como los de las películas o la televisión), los niños piensan que al final de las quince sesiones van a tener como resultado cajas de lata con ojos que hacen tareas, que limpian la casa, que se mueven de manera extraña, que reparan artefactos dañados y que hablan, entre otras habilidades.

Con este panorama queda abierta la pregunta de si al final del club este objetivo es alcanzado por los participantes o no. Podría pensarse que si esas son las expectativas del público, de alguna manera Maloka tendría que cumplirlas, de no ser así, ¿cómo es posible que después de dos años los niños sigan yendo y quedándose en este espacio de manejo del tiempo libre? (Cremers, 2003: 23). Frente a esta pregunta la posición de los clubes de tecnología es de doble cara. Por un lado, frente a la robótica y los imaginarios que de este universo reproducen los medios, los clubes se asumen como un escenario de divulgación tecnológica y validación en términos de información en el que los niños tienen la

\footnotetext{
${ }^{6}$ Estas siglas corresponden a robots de la pelicula Star Wars.
}

oportunidad de disfrutar reconociendo el mundo que les rodea; y por otro, de manera complementaria a ésta, una posición que está relacionada con su público, sus expectativas y el contexto en el que estamos, es decir lo que significa e implica (en términos de desarrollo apropiado, apropiación social de la tecnología y experiencia del público) que niños se acerquen al mundo de la tecnología de una manera distinta; esta posición es de orden pedagógico.

Con relación a lo anterior, los clubes de tecnologia han desarrollado una propuesta metodológica que tiene un objetivo doble. En primera instancia, motivar y hacer posible que los niños se acerquen al mundo de la robótica desde la creación (Rodríguez, 1996: $6)$; en segundo lugar, promover la apropiación de sentido crítico sobre dichas creaciones desde un ejercicio de reflexión continuo, el cual abarca a participantes y facilitadores (Ghiso, 2000: 5 y 7 ).

\section{EL GOCE COMO DESCUBRIMIENTO}

Uno de los pilares fundamentales de la propuesta metodológica de los clubes de tecnología es la motivación para crear, entendida ésta como la generación de un ambiente propicio para que los niños vean posible la robótica, ya no desde el universo mediático, sino desde su cotidianidad tecnológica. Con relación a lo anterior, la experiencia que viven los participantes está caracterizada por una continua exploración de los referentes tecnológicos que hacen parte de su contexto (McLaren, 1998: 75). Esta estrategia es concebida como un mecanismo que permite a los participantes descubrir una nueva manera de interpretar el mundo y de descubrirse en ese proceso, aspectos que son característicos del goce propio del juego (Bautista-Vallejo, 2002: 3-4). EI siguiente fragmento recoge un poco esta idea de goce y descubrimiento, en la voz de una niña que fue parte del club durante 2005:
De las cosas que más me gustaron fue cuando en la primera sesión des cubri que habia diferentes tipos de robots... yo pensaba que los robots eran todos con forma de humano, como los de las películas, pero ese dia me di cuenta que habia robots, médicos, con formas de animales, que servian para explorar... me emocioné tanto que me dieron muchas ganas de experimentar... de saber más, de conocer más... (Niña de 14 años. Lleva seis meses en el club).

De acuerdo con esto, el ejercicio de exploración propuesto hace posible que los niños, por un lado, aprehendan los componentes propios de la robótica, relacionándolos y asociándolos con lo que conocen y, por otro, que vayan complejizando su visión del mundo y, en particular, de su universo tecnológico.

Un ejemplo de esto es cuando se presenta el concepto de "sistema" a los niños. Este concepto que permite entender la robótica como una integración de diferentes disciplinas que cumplen tareas específicas articuladas a un fin, es introducido a partir de la asociación cotidiana de cómo operan los electrodomésticos y las alarmas caseras, lo que esto se logra a través de preguntas abiertas y dirigidas que buscan que ellos mismos den sentido al concepto de sistema desde lo que saben y conocen (Geslin, 2003: 26). Otro ejemplo es la aproximación al concepto de "fuentes de energía" que permiten que un robot funcione, descubriendo con los niños las fuentes de energía que normalmente se utilizan en sus hogares: la electricidad, las baterías y el sol, entre muchas otras; por otra parte, para desarrollar la noción de lógica binaria, consideramos la manera en la que ellos toman decisiones a diario, comparable a la manera como se logra que un robot actúe (tome decisiones) frente a un propósito predeterminado.

Descubrir que el mundo de la robótica es cercano es el punto de partida para la creación, segundo pilar fundamental de la propuesta metodológica. 


\section{EL GOCE COMO CREACIÓN}

El sentido de creación que guía los clubes de robótica está ligado a dos momentos complementarios: por un lado la puesta a prueba y experimentación de los descubrimientos realizados en la etapa de motivación, como un mecanismo para entender su funcionamiento y, asi mismo, complejizar su sentido tecnológico (Rodríguez, 1996: 5); y por otro, la utilización de esa idea o referente complejizado para la elaboración de un primer robot.

En este sentido, la apuesta detrás de la creación de un robot, es decir, la posibilidad de que el niño participante alcance sus expectativas, se ha construido alrededor de la complejización de sus referentes tecnológicos a la luz de la experimentación y la prueba. En otras palabras, para crear es necesario pasar de ver el mundo con otros ojos, a poner en juego ese descubrimiento; a que esas asociaciones cotidianas hechas pasen de ser una relación mental del niño con su mundo, a ser aprehendidas por él como realidades

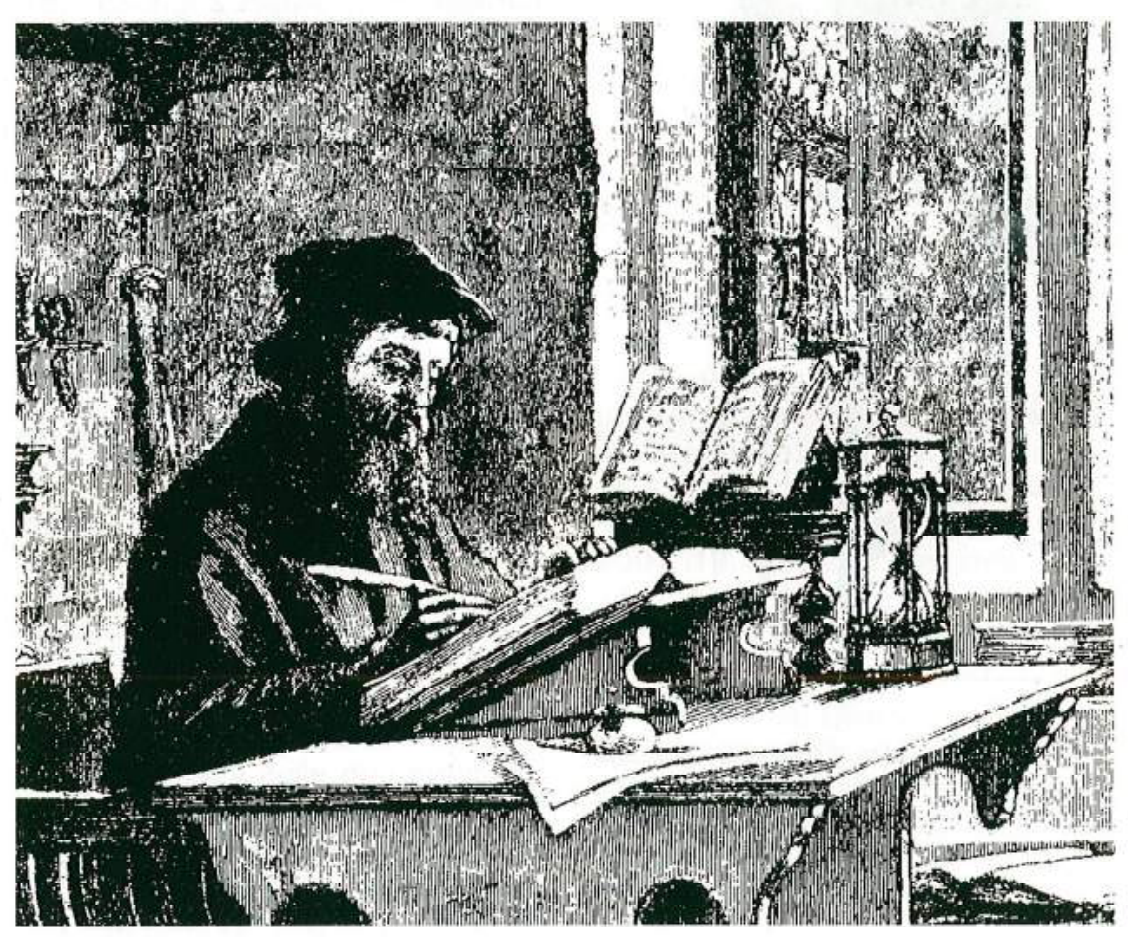

concretas y manipulables, a que sus posibilidades tecnológicas de uso y aplicación en el mundo real (más allá de la pantalla) sean experimentadas con miras a la comprensión, para luego considerar antes de hacer, o mejor, como una manera de preparar el hacer, de definir cuál es el uso y aplicación que estos nuevos referentes tecnológicos tienen en la imaginación de los participantes (Martín-Barbero, 2001: 9). El ejercicio de ponerse en contacto con herramientas y de manipular objetos es también caracteristico de la actividad lúdica, ya que a través de este tipo de dinámicas, el niño construye un nexo nuevo con las cosas que le permite integrarse socialmente con su entorno (Jiménez, 1998: 12), lo que responde a la lógica de resignificación del mundo, en este caso del universo tecnológico que se ha mencionado.

Un ejemplo útil de lo expuesto es el ejercicio de explorar, contextualizar, experimentar y aplicar los sensores que se desarrollan con los niños del club. Este ejercicio consiste en aproximarse al concepto de sen- sores, primero desde la idea de sentidos, de manera particular en los animales en quienes es más visible el potencial que tienen para percibir el mundo, y luego desde las alarmas. Posterior a la aproximación, viene la experimentación con sensores ópticos reales sobre texturas distintas: distancias, ángulos y superficies de diversos colores y formas, entre otros. Este mirar para ver cómo funciona se convierte en un ejercicio lúdico que tiene como trasfondo la complejización del referente sensorial del reino animal, y la comprensión más amplia de un universo tecnológico (que abarca la cotidianidad desde nuevas perspectivas). Luego del ejercicio el niño ve su mundo de otra forma: las alarmas esconden un mecanismo ya no desconocido y los animales son alegorias de pequeños carros que se mueven, paran, o giran dependiendo de a qué se estén enfrentando.

Esta nueva asociación desde la experiencia del universo tecnológico deja al niño en condiciones de preguntarse ¿qué puedo hacer con esto?, ¿cómo puedo usarlo para hacer un robot?, o ¿qué quiero que mi robot haga? Es en este proceso de aplicar los conceptos trabajados desde la experimentación que el niño empieza a armar, a modo de rompecabezas, lo que va a ser su primer robot. $Y$ es alli también que sus expectativas comienzan a ser una realidad. (Cremers, 2003: 26).

\section{PENSAR, JUGAR, APLICAR: ¿ENTENDER? EL GOCE COMO UNA HERRAMIENTA PARA LA REFLEXIÓN}

Como dijimos anteriormente, junto al acercamiento de los niños al mundo de la robótica desde la creación, el otro objetivo fundamental de los clubes de tecnología es promover la apropiación del sentido crítico sobre dichas creaciones, desde un ejercicio de reflexión continuo, el cual abarca a participantes y facilitadores (Ghiso, 2000: 7). 
Ahora bien, la reflexión como resultado de un proceso de exploración de referentes y la experimentación y complejización de los mismos es también un ejercicio creativo que permite a niños entender su proceso de aprendizaje y darle sentido al universo de la robótica. Como diría Freire, este proceso de reflexión continua permite a los participantes tomar distancia mentalmente de su experiencia para poder visualizar lo vivido, formalizándolo, abarcándolo, "ensanchando la comprensión de los eslabones" que lo hacen posible (Freire, 1996: 12). En este sentido, el goce de entender el mundo y de participar en el proceso de explicarlo es viable gracias a un ejercicio de acompañamiento continuo por parte de los tutores, que tiene como base fundamental el diálogo (McLaren, 1998: 78-80). El siguiente comentario nos centra sobre este argumento desde la voz de un tutor del club quien reflexiona sobre su relación con los niños:

Era curioso ver como ambos estábamos encima de la mesa y nos sorprendiamos a la vez cuando la cosa funcionaba, y cuando no funcionaba, ambos identificábamos los errores, en diferentes niveles, pero igual... son experiencias paralelas. Siempre hay espacio para la sorpresa. (Ingeniero electrónico recién graduado. 21 años. Lleva dos años en el club).

La comunicación que entablan los tutores con los niños sobre su experiencia, tiene como objetivo facilitar el establecimiento de relaciones evidentes entre los referentes de la cotidianidad tecnológica y entre los ejercicios prácticos que se realizan para probar cómo funciona el universo de la robótica. Es importante resaltar que el objeto de este diálogo es lo vivido en las sesiones, es decir, lo explorado, experimentado y construido por los niños (McLaren, 1998). Lo que se busca develar conjuntamente entonces, es la forma cómo se hicieron las cosas, lo que se pensaba sobre el universo tecnológico antes de la sesión y la manera cómo ese "pensar sobre" se ensancha en ese proceso de creación. Ese engrande- cer la visión del mundo comprende no sólo el funcionamiento de la robótica, el descubrimiento por parte de los niños de las cajas negras que están detrás de los mecanismos que hacen que los robots funcionen, así como el descubrimiento de que ellos pueden hacer un robot, sino también, y sobre todo, el descubrimiento de que la tecnologia tiene un contexto y responde a unas necesidades concretas y locales ya no sólo subjetivas ${ }^{7}$.

Es en este punto cuando el goce por crear un robot se mezcla con el goce que produce entender cómo funciona el mundo, y es allí cuando la misión de apropiación social de la tecnología se alcanza abarcando los intereses y expectativas particulares de los participantes, e incluso llegando a superarlos.

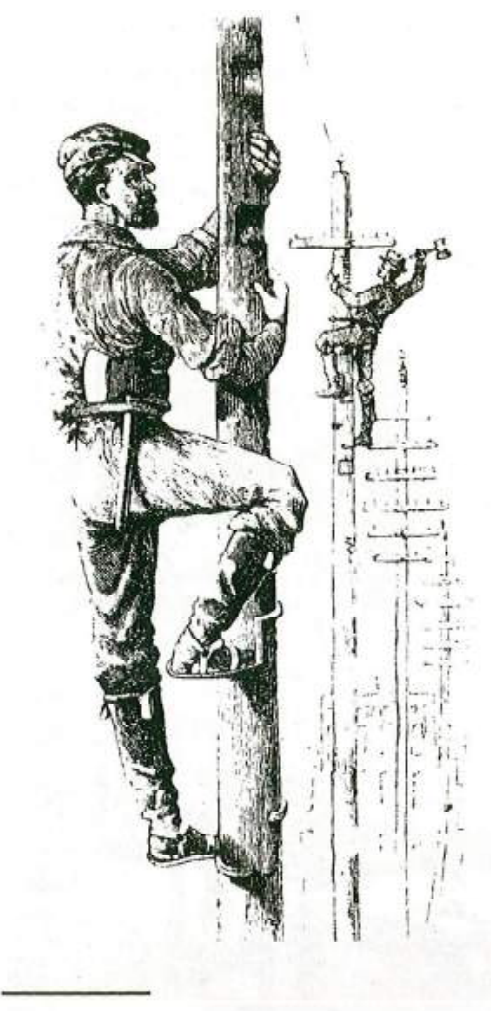

${ }^{7} \mathrm{El}$ aprender por aprender es propio de un goce individual que refleja la sociedad de consumo, en este caso hablamos de un goce de entender el mundo en compañía, que está encaminado, en últimas, a fortalecer procesos de identidad cultural.

\section{EL PAPEL DEL ACOMPAÑAMIENTO O EL GOCE DE ACOMPAÑAR}

\begin{abstract}
Ahora con el trabajo de los clubes he empezado a diseñar cosas para los chicos, yo me he dado cuenta que ellos piensan cosas rarísimas, lo piensan no desde lo posible, sino desde lo que quieren hacer... entonces yo diseño cosas para que juntos podamos hacer que esas cosas que piensan se vuelvan posibles y reales... eso me parece súper bacano. Yo aprendo mucho de ellos, las mismas cosas que estoy diseñando con ellos, yo mismo les encuentro aplicación en los proyectos que tengo que hacer para la universidad... eso hace que cuando los proyectos de cada uno resultan todos nos emocionemos y nos pongamos contentos... (Estudiante de Ingenieria mecatrónica. 20 años. Lleva un año en el club).
\end{abstract}

Como dijimos anteriormente, el proceso vivido por los niños, es posible gracias al acompañamiento de los tutores. Ahora bien, este acompañamiento es también un ejercicio de descubrimiento del mundo para quienes acompañan, y se da gracias a su continua interlocución con los participantes (Geslin, 2003: 25-26). En este sentido, la participación de los tutores en el club, su papel de construir el material de apoyo, de explorar junto con los niños los referentes cotidianos de la tecnología, de apoyarlos en sus ejercicios de experimentación de esos referentes, y en el ejercicio continuo de reflexionar con ellos sobre dónde están y por qué están ahi, durante la sesión y posterior a ella, permite a los tutores vislumbrar la dimensión de contexto de lo tecnológico, una dimensión social apropiada y pertinente (Ghiso, 2002: 8).

Cuando el tutor participa de la actividad lúdica no sólo como facilitador, sino como otro participante más, al mismo nivel que los niños, cuando descubre con ellos y se sorprende con ellos, de algún modo también está disfrutando, esto hace que la distancia generacional y disciplinar se rompa y el diálogo se haga po- 
sible, o, en otras palabras, según Maturana, el goce propio del juego se transforma en una experiencia cultural más que una actividad didáctica en el cual "el otro surge como otro legitimo" (Maturana citado en Jiménez, 1998: 38). La apuesta no es entonces transmitir lo que el tutor sabe -como futuro ingeniero- a unos niños que no saben sobre tecnología en términos conceptuales o técnicos, pero que son muy curiosos (Hoffmann, 2001: 92-93). Por el contrario, la apuesta parte del reconocimiento de que existen saberes complementarios de lado y lado, saberes sobre cómo funcionan las cosas y saberes sobre cómo usarlas, saberes sobre cómo se relaciona lo tecnológico con el mundo, y saberes sobre cómo necesito o quiero relacionar ese conocimiento para entender mejor mi universo cotidiano. Desde esta perspectiva, el goce de dejarse sorprender mutuamente por las posibilidades que existen más allá de lo que ya sabemos es un goce compartido.

Sin embargo, es importante señalar en este punto que ese descubrir el mundo que se da de lado y lado es posible en la medida en que tanto niños como tutores se auto reconozcan como interlocutores válidos en el escenario de los clubes, lo cual es un proceso de socialización importante para ambos. Para los niños lo es en la medida en que se reconocen capaces de crear con otros y de ser participes de mundos posibles, en este caso el mundo de la robótica, que hasta el momento sólo existia a través de una pantalla; para los tutores, por su parte, como estudiantes universitarios de carreras tecnológicas, la socialización se genera cuando se reconocen como sujetos sociales en un escenario distinto al académico, como parte de una comunidad y con un papel constitutivo de la misma, en este caso el de ser responsables de acompañar el proceso de descubrimiento de otros a la luz de lo que saben; pero también esa socialización es posible cuando los tutores visualizan lo tecnológico desde esa lógica social, no de manera anecdótica, sino incluso con proyecciones personales hacia el futuro. En este sentido, la apuesta de los clubes de tecnologia se da también en el terreno de la formación de jóvenes como ciudadanos...

Yo no voy a desconocer el compromiso que tengo con el equipo, los objetivos que hay detrás de los clubes que le apuestan a poder trabajar con los niños de manera distinta... me siento parte de una \}apuesta más grande, somos muchos los que estamos preocupados porque la cosa funcione, no sólo los clubes... Yo estoy convencido de que estoy haciendo parte de un plan para conquistar el mundo. (Ingeniero electrónico recién graduado. 21 años Lleva dos años en el club).

\section{A MODO DE CONCLUSIÓN}

Si tuviéramos que concluir por alguna parte, quizás lo primero que habría que decir es que las estrategias generadas por Maloka para promover la apropiación social de la tecnología son estrategias que tienen como punto de partida el diálogo: el diálogo con las expectativas de los niños y el diálogo con el contexto; esta interlocución permanente ha hecho posible que la propuesta evolucione de manera permanente y dinámica.

Como decíamos al inicio de este documento, los escenarios culturales necesitan leerse y, por tanto, pensarse de manera distinta para atender a la demanda de consumo de entretenimiento en el que son encajados socialmente (Cremers, 2003: 19, 23 y 25). Decimos necesitan, pues de algún modo de esto depende su supervivencia. Esta lectura, sin embargo, requiere de una visión institucional clara desde la cual sea evidente que cumplir las expectativas de diversión de su público hace parte de las estrategias de divulgación y apropiación social de la tecnología, la ciencia, el arte, el patrimonio histórico, o la que fuese la misión particular de cada museo, que sean apropiadas a su contexto y no sólo al contexto de la sociedad de consumo.

En este sentido, si pensamos esto en términos de apropiación tecnológica es necesario entender el caso colombiano, asi como el de muchos países latinoamericanos, en el marco de un pais con una naturaleza subsidiaria frente a la tecnologia, es decir, que su acercamiento a ella se hace desde el uso y no desde la producción ni el desarrollo, lo que de alguna manera genera relaciones de dependencia económica y de alienación frente a las propias necesidades; ahora bien, sin que esta realidad sea natural a nuestro contexto, vale la pena señalar que hace parte de una estructura geopolíticas que es reforzada desde los medios de comunicación. La misión de Maloka frente a esta perspectiva no es reproducir esta dinámica, sino generar un espacio de posibilidad para la creación en el que la tecnologia pueda ser vista de forma crítica y apropiada. Lo que se constituye en sí mismo en un mecanismo de identidad cultural para que su público se reconozca en el mundo, con y desde otras competencias.

Por último, por tener como punto de partida el contexto y en particular el reconocimiento de sus características, pero también de sus limitaciones y necesidades, la propuesta que sustenta a los clubes de tecnología de Maloka se encuentra en continua evolución, es decir, puede cambiar en la medida en que el contexto se transforma. Esto explica que la exploración de referentes de la cotidianidad tecnológica en el que se sumergen los participantes sea un pilar fundamental de los clubes. Así, si entendemos a Maloka en un contexto constituido por múltiples caras sociales, económicas y culturales, habrá que entender que el goce de descubrir el mundo es posible siempre y cuando la propuesta sea capaz de reconocer esa diversidad desde el punto de vista tecnológico. 


\section{BIBLIOGRAFÍA}

Aubud, L., J. S.: Escobar, R., A. L. y Rojas P. (2005). De la percepción a la apropiación social de la ciencia y la tecnología. En: Colciencias (2005). La percepción que tienen los colombianos sobre la ciencia y la tecnología. Bogotá: Colciencias.

Bautista-Vallejo, J. y López, N. (2002). El juego didáctico como estrategia de atención a la diversidad. En: Revista Ágora Digital. N. ${ }^{\circ}$ 4. Extraido de: http://www. uhu. es/agora/digital/numeros/numeros _ppal.htm

Cremers, F. (2003). La importancia de divertirse en los museos: sobre las dificiles relaciones entre el museo y sus visitantes. En: XANTHOUDAKI, M. (Ed.) Un lugar para descubrir: la enseñanza de las ciencias y la tecnología en los museos. Milán: T\&T Studio, San Donato Mila.se.

Freire, P. (1996). Cartas a Cristina, reflexiones sobre mi vida y mi trabajo. México: Siglo Veintiuno Editores.

Gagliard, A. (2005, Julio 18). Los museos y el público. Extraido el 27 de julio de 2005, de: www.museosdevenezuela. org/documentos/3publicos/museosypublico003. shtml

GESLIN. P. (2003). Las formas de apropiación de los objetos técnicos o el paradigma antropotecnológico. En: BUENo, C y
Santos, M. J. (Coord.). Nuevas Tecnologias y cultura. México: Anthropos.

Gisso, A. (2000). Potenciando la diversidad, diálogo de saberes una práctica hermenéutica colectiva. Extraído el 21 de agosto de 2005, de: http://biblioteca digital.conevyt.org.mx/

Giddens, A. (1994). Consecuencia de la modernidad. Madrid: Alianza.

Hoffmann, R. (2001). La intuición científica y la imaginación. En: CHIMAL, C. Luz Interior: conversaciones sobre ciencia y literatura. México: Tusquets Editores, Metatemas.

Jiménez, C. (1998). Pedagogía de la creatividad y de la lúdica. Emociones, inteligencias y habilidades secretas. Bogotá: Editorial Mesa redonda-Magisterio.

Martin-Barbero, J. (2001). Políticas culturales de nación en tiempos de globalización. En: Revista Gaceta. N. ${ }^{\circ} 48$. Bogotá: Ministerio de Cultura. pp. 1-19.

(2002). Jóvenes: comunicación e identidad. En: Revista de cultura Pensar Iberoamérica. N. ${ }^{\circ} 0$. Extraido el 13 de mayo de 2005, de: http://www. campus-oei.org/pensariberoamerica/ ric00a03.htm

MCLAREN, P. (1998). Desde los márgenes: geografías de la identidad, la pedagogía y el poder. En: McLAREN. Pedagogía, identidad y poder. Los educadores frente al multiculturalismo. Rosario, Argentina: Editorial Homo Sapiens.

Medina, A. (2003). La cultura de la tecnociencia. En: BUENo, C. y SANTOS, M. J. (Coord.) Nuevas Tecnologias y cultura. México: Anthropos.

Mendia, R. (1991). Principios pedagógicos del tiempo libre. Corrientes de pensamiento. En: Revista Infancia y sociedad. N. ${ }^{\circ}$ 8. 1991, marzo-abril. pp. 33-50. Extraído el 18 de septiembre de 2005, de: http://homepage.mac.com/rmendia/compartir_archivos/textos/monitoreducador IS199 18343350.pdf

RodRIGUez, A. (1996). La incorporación de un área de tecnología a la educación general En: Propuesta educativa. Flacso. Año 7. N. ${ }^{\circ}$ 15. 1996, diciembre. Extraido de: http://cab.cnea.gov.ar/gaet/ flacso/pdf

Sautino, C. Públicos y museos. Algunas reflexiones. Extraido el 11 de septiembre de 2005, de: www.museosdevenezuela. org/documentos/3publicos/museosypu blico007.shtml

SchmilchuK, G. (2005, 20 de agosto) Venturas y desventuras de los estudios de públicos. Extraido el 11 de septiembre de 2005, de: www.museosdevenezuela org/documentos/3publicos/museosypubli co006_1.shtml 